\title{
Improved diffusion Monte Carlo propagators for bosonic systems using Itô calculus
}

\author{
P. Håkansson and M. Mella ${ }^{a}$ \\ School of Chemistry, Cardiff University, Main Building, Park Place, Cardiff CF10 3AT, United Kingdom \\ Dario Bressanini, Gabriele Morosi, and Marta Patrone \\ Dipartimento di Scienze Chimiche ed Ambientali, Universita' dell'Insubria, Sede di Como, via Lucini 3, \\ 22100 Como, Italy
}

(Received 17 July 2006; accepted 29 September 2006; published online 10 November 2006)

\begin{abstract}
The construction of importance sampled diffusion Monte Carlo (DMC) schemes accurate to second order in the time step is discussed. A central aspect in obtaining efficient second order schemes is the numerical solution of the stochastic differential equation (SDE) associated with the Fokker-Plank equation responsible for the importance sampling procedure. In this work, stochastic predictor-corrector schemes solving the SDE and consistent with Itô calculus are used in DMC simulations of helium clusters. These schemes are numerically compared with alternative algorithms obtained by splitting the Fokker-Plank operator, an approach that we analyze using the analytical tools provided by Itô calculus. The numerical results show that predictor-corrector methods are indeed accurate to second order in the time step and that they present a smaller time step bias and a better efficiency than second order split-operator derived schemes when computing ensemble averages for bosonic systems. The possible extension of the predictor-corrector methods to higher orders is also discussed. (C) 2006 American Institute of Physics. [DOI: 10.1063/1.2371077]
\end{abstract}

\section{INTRODUCTION}

In the study of low-temperature boson systems such as rare gas clusters, the diffusion Monte Carlo (DMC) method ${ }^{1}$ appears as an attractive computational complement to experimental techniques due to its ability of exactly solving the Schrödinger equation for these species. In brief, DMC usually contains two key components, namely, an importance sampled diffusion of points in configurational space (i.e., the diffusing walkers are guided toward important regions of space by means of an approximate wave function $\psi_{T}$ ) and a branching process that changes the local number of walkers according to a specified weight. Whereas importance sampling greatly reduces the statistical error in DMC, the branching process makes the asymptotic distribution approach the exact ground state (in the limit of small time step $\tau \rightarrow 0$ ) for bosonic systems.

It can be shown that the introduction of the trial wave function $\psi_{T}$ as a guide for the diffusion is equivalent to writing the modified Schrödinger equation ${ }^{2}$ as follows:

$$
\begin{aligned}
-\frac{\partial f(x, t)}{\partial t}= & \underbrace{-\frac{1}{2} \nabla^{T} D \nabla f(x, t)+D \nabla \cdot[F(x) f(x, t)]}_{=L f(x, t)} \\
& +E_{L}(x) f(x, t),
\end{aligned}
$$

where $f(x, t)=\psi_{0} \psi_{T},{ }^{3} E_{L}(x)=\psi_{T}(x)^{-1} H \psi_{T}(x)$ is the local energy, $H$ is the $N$-dimensional Hamiltonian, $t$ is the imaginary time, $F(x)=\psi_{T}(x)^{-1} \nabla \psi_{T}(x)$ is the quantum force, and $D$ is a diagonal diffusion matrix. The operator $L$ acting on $f(x, t)$ is

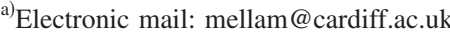

the Fokker-Planck (Schmoluchowski) operator, whose stationary solution is $\psi_{T}^{2}(x)$ and is the one responsible for the importance sampling during a DMC simulation.

Formally, the solution of the modified Schrödinger equation [Eq. (1)] can be obtained by iterating the action of the projection operator $\exp \left[-\tau\left(L+E_{L}\right)\right]$ over an initial distribution $f(x, 0)$ until convergence to the ground state is reached:

$$
\lim _{n \rightarrow \infty}\left[e^{-\tau\left(L+E_{L}\right)}\right]^{n} f(x, 0) \rightarrow \psi_{T}(x) \psi_{0}(x),
$$

where, obviously, $t=n \tau$ provides the connection with Eq. (1). This formal equation is, however, not particularly useful because it assumes that either an analytical representation in configuration space of $\exp \left[-\tau\left(L+E_{L}\right)\right]$ or a numerical scheme capable of producing identical results is known. In practice, having the exact analytical form of the projector $\exp \left[-\tau\left(L+E_{L}\right)\right]$ is equivalent to knowing all the eigenstates and eigenvalues of Eq. (1), which is clearly an extremely difficult task to be tackled for many-body systems. As a consequence, the theory of DMC relies on the possibility of writing an accurate approximation for the projector presenting a systematic error that can be made as small as needed by selecting appropriately the parameter $\tau$, i.e., the time step. One such approximation is provided by the following symmetric splitting of the evolution operator:

$$
e^{-\tau\left(L+E_{L}\right)} \simeq e^{-(\tau / 2) E_{L}} e^{-\tau L} e^{-(\tau / 2) E_{L}},
$$

which is known to be formally second order in $\tau^{4}$. If the action of the two operators $e^{-\tau L}$ and $e^{-\tau E_{L}}$ was exactly known, or if it was possible to simulate it numerically with some exact scheme, the systematic (time step) error for the mean value of a local property $O_{L}(x)=\psi_{T}(x)^{-1} \mathcal{O} \psi_{T}(x)$ over 
the limiting distribution $f(x, \infty)$ would be guaranteed to be second order for $\tau$ small enough. ${ }^{5}$ Unfortunately, whereas the exact simulation of $e^{-\tau E_{L}}$ does not represent a problem, the latter being usually treated as a weighting operator, the situation for $e^{-\tau L}$ is made far more complicated by the impossibility of reproducing exactly its action for a general manybody system. As a consequence, it becomes necessary to approximate the effect of $e^{-\tau L}$ with some numerical method, thus introducing additional errors in the representation of the exact $e^{-\tau\left[L+E_{L}\right]}$ besides neglecting a double commutator implicit in Eq. (3).

The effect of the Fokker-Planck operator driving the distribution of points toward the stationary solution of the Fokker-Planck equation $L f(x)=0$ [with $L$ shown in Eq. (1)] is often simulated by solving the approximate stochastic differential equation (SDE) (hereafter Euler scheme) as follows: ${ }^{2}$

$$
Y_{n+1}^{(k)}=Y_{n}^{(k)}+D^{(k, k)} F^{(k)}\left(Y_{n}\right) \tau+b^{(k, k)} \Delta W_{n}^{(k)},
$$

where $Y_{n}^{(k)}$ is the $k$ th coordinate of the vector representing the position in space of all particles in the system after $n$ simulation steps, $F^{(k)}\left(Y_{n}\right)$ is the $k$ th component of the quantum force at $Y_{n}, \tau=t_{n+1}-t_{n}$ is the simulation time step, $\Delta W^{k}$ is an $N(0, \tau)$ Gaussian random number with zero average and variance equal to $\tau$, and $\left(b b^{T}\right)^{(k, k)}=D^{(k, k)}$ is the $k$ th diagonal element of the diffusion matrix in Eq. (1). This is a finite-step approximation of the exact SDE sampling $f(x)=\psi_{T}^{2}(x)$,

$$
X_{t}^{(k)}=X_{t_{0}}^{(k)}+D^{(k, k)} \int_{t_{0}}^{t} F^{(k)}\left(X_{s}\right) d s+b^{(k, k)} \int_{t_{0}}^{t} d W_{s}^{(k)},
$$

with $W_{t}$ being a Brownian motion in $\mathbb{R}^{N}$ (at time $t$ ). In this respect, it has recently been recognized that the accurate solution of Eq. (5) is crucial in obtaining more efficient DMC schemes, ${ }^{6,7}$ a finding that led Chin $^{6}$ to propose improved short time approximations for the Fokker-Planck propagator $e^{-\tau L}$ based on the use of exponential splitting formulas, ${ }^{4}$ effectively overcoming some of the limitations implicit in Eq. (4). In his original work, Chin proposed two alternative exponential splittings (LGV2a and LGV2b defined in the following) to solve Eq. (5), letting the form of these more accurate representations for $e^{-\tau L}$ suggest the structure of the numerical algorithm to solve the SDE. In this way, numerical schemes giving ensemble averages accurate, at least, to second order in $\tau$ were derived, ${ }^{6}$ an important improvement over Eq. (4) which is only accurate to first order in $\tau$. Besides, the LGV2a algorithm proposed in Ref. 6 was rewritten in a less canonical way (dubbed LGV2c), providing an alternative derivation of another scheme proposed previously by Helfand $^{8}$ and tested by Anderson by simulating the electronic structure of $\mathrm{H}$ and $\mathrm{He}{ }^{9}$

In the context of DMC simulations, the availability of a second order algorithm to simulate $e^{-\tau L}$ means that the symmetric splittings presented in Eq. (3) become second order not only formally but also in practice. These, in turn, may present a reduced time step bias, so that, given a maximum acceptable error for the system properties, one may be allowed to use a larger time step making the simulation far more efficient. In a similar way, higher order (e.g., fourth) factorizations of the evolution operators [i.e., $e^{-\tau L}$ or $\left.e^{-\tau\left(L+E_{L}\right)}\right]$ are known to provide even smaller time step biases than Eq. (4) or second order algorithms. ${ }^{7,10}$ This improvement, however, comes at the cost of computing $F(x)$ many more times and requires the additional calculation of potential and local energy gradients.

In spite of their improved performance, the increased cost per step featured by high order algorithms suggests that it may be advantageous to seek better second order approximations to the DMC evolution operator within the framework provided by Eq. (3). Since the effect of $e^{-(\tau / 2) E_{L}}$ is simulated exactly by the branching step, the overall task is reduced to finding a better numerical scheme simulating the effect of the Fokker-Planck operator. In this work, we address the issue of solving Eq. (5) by seeking more accurate numerical schemes with the mathematical theory (Itô calculus) specifically developed for SDE's. ${ }^{11}$ This gives us an alternative approach to the one provided in Ref. 6, where a similar task was carried out by working at the Fokker-Planck equation (FPE) level rather than at the SDE level as we propose. Despite this difference, it is, of course, assured that the ensemble averages obtained using SDE theory will correspond to the properties of the Fokker-Planck equation. ${ }^{11}$ In this respect, the net advantage provided by using Itô calculus is represented by the knowledge of precise mathematical conditions that need to be fulfilled by the numerical scheme to present a given order of convergence with respect to $\tau$. In other words, it would allow us to prove the order in $\tau$ of the computed ensemble averages. ${ }^{12}$

At this stage, it must be stressed that the convergence toward the solution of a SDE could be measured in two ways. First, there is one indicated as "strong" convergence, where an accurate approximation for the trajectory solving the SDE is sought. Second, there is "weak" convergence, where only accurate distributions are desired and which is easier to obtain. Luckily, in DMC the interest lies on the ensemble average of first or higher moments of a local property $O_{L}(x)$, these quantities relying only on the properties of the weak convergence. As a consequence of these arguments, we are therefore allowed to use the weak Itô-Taylor expansion $^{12}$ as starting point for the construction of numerical schemes approximating Eq. (5), and we review briefly this mathematical tool in the next section to provide the reader with a self-containing discussion. Following this introduction, two numerical schemes presented in Refs. 12 and 13 solving Eq. (5) with second order accuracy in $\tau$ are revised, and it is illustrated how we can confirm the consistency of these algorithms with theory. ${ }^{12}$ Generally speaking, these schemes belong to the categories of "explicit second order" and "predictor-corrector" approaches specifically developed to solve SDE's, the heuristic application of predictor-corrector methods borrowed from the theory of ordinary differential equations usually leading to poor performances. ${ }^{14}$ To our best knowledge, no previous attempt of testing the performances of these two families in DMC simulations of bosonic clusters has been presented in the literature. Two alternative second order schemes provided in Ref. 6 are also investigated in the framework of Itô calculus, proving that they are consistent with the weak Itô-Taylor 
expansion. Finally, we show the results of numerical simulations on model systems and doped helium clusters obtained employing the presented second order algorithms. The discussion of these results provides evidences that the predictorcorrector schemes derived from the weak Itô-Taylor expansion are more accurate and efficient than split-operator based ones, facilitating their application in different contexts as well as possible algorithmic extensions. We also provide strong arguments indicating that it is advantageous to substitute the widely used DMC employing an acceptance/ rejection step with the predictor-corrector scheme.

\section{SECOND-ORDER NUMERICAL SCHEME}

The theory used to develop high order weak Itô-Taylor expansions is well covered in the literature. ${ }^{12}$ Thus, this section will only review the approach to obtain a second order weak Itô-Taylor numerical scheme providing some of the details in Appendix A, and we refer the reader to Ref. 12 for further details. As starting point, one expresses the integrand $F\left(X_{s}\right)$ in Eq. (5) using the Itô formula ${ }^{15}$ such that the $k$ th component of $F\left(X_{s}\right)$ is

$$
\begin{aligned}
F^{(k)}\left(X_{s}\right)= & F^{(k)}\left(X_{t_{0}}\right)+\int_{0}^{s} L^{(0)} F^{(k)}\left(X_{z}\right) d z \\
& +\sum_{j=1}^{N} \int_{t_{0}}^{s} L^{(j)} F^{(k)}\left(X_{z}\right) d W_{z}^{(j)},
\end{aligned}
$$

where the operators are

$$
\begin{aligned}
& L^{(0)}=\frac{\partial}{\partial t}+\sum_{i=1}^{N} D^{(i, i)} F^{(i)} \frac{\partial}{\partial x^{(i)}}+\frac{1}{2} \sum_{i=1}^{N} D^{(i, i)} \frac{\partial^{2}}{\partial x^{(i)^{2}}}, \\
& L^{(j)}=b^{(j, j)} \frac{\partial}{\partial x^{(j)}}
\end{aligned}
$$

for the case of a constant diagonal diffusion matrix $b$. With the integrand of Eq. (5) expanded with the Itô formula, we demonstrate in Appendix A the derivation of the simplified second order weak Itô-Taylor scheme as follows:

$$
\begin{aligned}
Y_{n+1}^{(k)}= & Y_{n}^{(k)}+D^{(k, k)}\left(F^{(k)}\left(Y_{n}\right) \tau_{n}+\frac{1}{2} L^{(0)} F^{(k)}\left(Y_{n}\right) \tau_{n}^{2}\right. \\
& \left.+\sum_{j=1}^{N} L^{(j)} F^{(k)}\left(Y_{n}\right) \frac{1}{2} \tau_{n} \Delta W_{n}^{(j)}\right)+b^{(k, k)} \Delta W_{n}^{(k)} .
\end{aligned}
$$

The weak Itô-Taylor scheme is central to this work and is a valuable starting point due to its mathematically proved order of convergence and the fact that alternative numerical schemes can be tested against Eq. (9) as discussed in the following.

The proof of order $\beta$ convergence for a weak scheme such as Eq. (9) is provided by theorem 14.5.2 in Ref. 12 [e.g., it is proved that $\beta=1$ and $\beta=2$ for Eqs. (4) and (A3), respectively]. Theorem 14.5 .2 requires $F^{(k)}(X)$ to fulfill the Lipschitz condition and certain smoothness conditions, and that all multiple integrals of the proper order are included. In our specific case, this means that for $\beta=2$, only single and double but no triple integrals present in the weak Itô-Taylor expansion should be included [cf. Eq. (A3)]. In particular, the theorem states that the error for a computed expectation value with the second order weak Taylor scheme in Eq. (9) satisfies

$$
\left|\left\langle g\left(X_{T}\right)-g\left(Y_{T}^{(\tau)}\right)\right\rangle\right| \leqslant C_{g} \tau_{\max }^{2},
$$

where \langle\rangle is the expectation value of the difference between the values assumed by a test function $g(\cdot)$ over points in space visited by the exact process $X_{T}$ and by the process $Y_{T}^{(\tau)}$ simulated with Eq. (9), and $\tau_{\max }$ is the largest time step used in the interval of integration $[0, T]$.

It is important to note that derivatives of the quantum force (the drift in SDE language) are present in Eq. (9), and this is rather inconvenient for the sake of numerical simulations because the number and complexity of the required terms increase quickly with the system size. As a consequence, the development of schemes alternative to the weak Itô-Taylor expansion becomes a worthy exercise. In this respect, it is useful to remind that, given a single step scheme $Y$ proved to be of order $\beta$ [cf. Eq. (9) for order 2] and an alternative scheme $\hat{Y}^{(\tau)}$, the latter is also of order $\beta$ if it fulfills the condition

$$
\begin{aligned}
& \left|\left\langle\prod_{j=1}^{l}\left\{\hat{Y}^{\left(i_{j}\right)}-y^{\left(i_{j}\right)}\right\}-\prod_{j=1}^{l}\left\{Y^{\left(i_{j}\right)}-y^{\left(i_{j}\right)}\right\}\right\rangle\right| \\
& \quad \leqslant K(y) \tau^{\beta+1}, \quad i_{j}=1, \ldots, N, \quad l=1, \ldots, 2 \beta+1,
\end{aligned}
$$

where $x^{(i)}$ denotes the $i$ th component of vector $\mathbf{x}, K(y)$ is limited to polynomial growth, $N$ is the dimensionality, and the same initial position $y$ is used in each of the two products in Eq. (11). ${ }^{16}$ In this case, $\hat{Y}^{(\tau)}$ is said to be $\beta$ equivalent to $Y$. Equation (11) can be used to derive alternative, derivativefree higher order schemes as done in Refs. 12 and 16 or, as used below, to examine already proposed schemes.

\section{DERIVATIVE-FREE SCHEMES FOR SDE}

We start this section by considering two derivative-free approaches proposed in Ref. 13, simplifying them for the case of a constant diagonal diffusion matrix. These algorithms are the explicit second order scheme (E2)

$$
Y_{n+1}^{(k)}=Y_{n}^{(k)}+D^{(k, k)} \frac{1}{2}\left\{F^{(k)}\left(Y_{n}\right)+F^{(k)}\left(\tilde{Y}_{n+1}\right)\right\} \tau_{n}+b^{(k, k)} \Delta W_{n}^{(k)},
$$

with

$$
\tilde{Y}_{n+1}^{(k)}=Y_{n}^{(k)}+D^{(k, k)} F^{(k)}\left(Y_{n}\right) \tau_{n}+b^{(k, k)} \Delta W_{n}^{(k)},
$$

and the second order predictor-corrector scheme (PC2), where the predictor $(\tilde{Y})$ is second order

$$
\begin{aligned}
& Y_{n+1}^{(k)}=Y_{n}^{(k)}+D^{(k, k)} \frac{1}{2}\left\{F^{(k)}\left(Y_{n}\right)+F^{(k)}\left(\tilde{\tilde{Y}}_{n+1}\right)\right\} \tau_{n}+b^{(k, k)} \Delta W_{n}^{(k)}, \\
& \tilde{\tilde{Y}}_{n+1}^{(k)}=Y_{n}^{(k)}+D^{(k, k)} \frac{1}{2}\left\{F^{(k)}\left(Y_{n}\right)+F^{(k)}\left(\tilde{Y}_{n+1}\right)\right\} \tau_{n}+b^{(k, k)} \Delta W_{n}^{(k)},
\end{aligned}
$$




$$
\tilde{Y}_{n+1}^{(k)}=Y_{n}^{(k)}+D^{(k, k)} F^{(k)}\left(Y_{n}\right) \tau_{n}+b^{(k, k)} \Delta W_{n}^{(k)}
$$

It is instructive to verify that E2 is indeed second order by expanding $F\left(\tilde{Y}_{n+1}\right)$ in Eq. (12) to first order using the Itô formula [cf. Eq. (6)]

$$
\begin{aligned}
F^{(k)}\left(\tilde{Y}_{n+1}\right)= & F^{(k)}\left(Y_{n}\right)+L^{(0)} F^{(k)}\left(Y_{n}\right) \tau+\sum_{j=1}^{N} L^{(j)} F^{(k)} \\
& \times\left(Y_{n}\right) \Delta W_{n}^{(j)}
\end{aligned}
$$

and inserting Eq. (17) into Eq. (12) to obtain

$$
\begin{aligned}
Y_{n+1}^{(k)}= & Y_{n}^{(k)}+D^{(k, k)}\left(F^{(k)}\left(Y_{n}\right) \tau+\frac{1}{2} L^{(0)} F^{(k)}\left(Y_{n}\right) \tau^{2}\right. \\
& \left.+\sum_{j=1}^{N} L^{(j)} F^{(k)}\left(Y_{n}\right) \frac{1}{2} \tau \Delta W_{n}^{(j)}\right)+b^{(k, k)} \Delta W_{n}^{(k)} .
\end{aligned}
$$

This shows that E2, rewritten as a single time step process, is consistent with Eq. (9). Using similar arguments, the scheme PC2 [cf. Eq. (14)] is also found to be consistent with Eq. (9).

At this point, few considerations on the stability of numerical schemes appear necessary to motivate the implementation and testing of $\mathrm{PC} 2$, which requires one more quantum force evaluation than E2. In the literature concerned with the numerical solution of deterministic ordinary differential equations, it is usually found that resorting to an implicit scheme where $Y_{n+1}$ is a function of both $Y_{n}$ and $Y_{n+1}$ do improve the stability of the solution with respect to the time step, with the net effect of producing acceptable solutions (i.e., with an error smaller that a chosen limit) on a wider range of time steps. ${ }^{17}$ The same issue was addressed also for SDE's in Ref. 13, where it was suggested that a predictorcorrector scheme may present improved performance and inherit some of the stability properties of the implicit stochastic counterpart [where $\tilde{\tilde{Y}}_{n+1}$ is replaced with $Y_{n+1}$ in Eq. (14)]. In our view, the possibility of finding improved stability when using PC2 is a strong motivation for its implementation and testing despite the additional cost.

Obviously, E2 and PC2 are not the only second order algorithms for SDE's present in the literature, the importance of simulating the Fokker-Planck equation having given impetus to the construction of alternative derivative-free schemes. The majority of the latter are based on the partitioning of the Fokker-Planck operator using a symmetric exponential splitting similar to Eq. (3). In this work, we are particularly interested in the performance of two second order algorithms (LGV2a and LGV2b) presented in Ref. 6, which are multistep algorithms with the following derivative-free form for LGV2a:

$$
\begin{aligned}
& \hat{Y}^{(k)}=Y_{n}^{(k)}+\frac{1}{2} D^{(k, k)} F^{(k)}\left(Y_{n}+\frac{1}{4} D F\left(Y_{n}\right) \tau\right) \tau, \\
& \hat{Y}=\hat{Y}^{(k)}+b^{(k, k)} \Delta W_{n}^{(k)},
\end{aligned}
$$

$$
Y_{n+1}^{(k)}=\hat{\hat{Y}}+\frac{1}{2} D^{(k, k)} F^{(k)}\left(\hat{\hat{Y}}+\frac{1}{4} D F(\hat{\hat{Y}}) \tau\right) \tau
$$

with $D F\left(y_{n}\right)$ indicating the matrix-vector product between $D$ and the quantum force, and for LGV2b:

$$
\begin{aligned}
& \hat{Y}^{(k)}=Y_{n}^{(k)}+b^{(k, k)} \widetilde{\Delta W}_{n}^{(k)}, \\
& \hat{\hat{Y}}=\hat{Y}^{(k)}+D^{(k, k)} F^{(k)}\left(\hat{Y}+\frac{1}{2} D F(\hat{Y}) \tau\right) \tau, \\
& Y_{n+1}=\hat{\hat{Y}}+b^{(k, k)} \widetilde{\Delta W_{n+1 / 2}^{\prime(k)},}
\end{aligned}
$$

where $\Delta \widetilde{W}^{(k)}$ and $\Delta \widetilde{W}^{\prime(k)}$ are $N(0, \tau / 2)$ random numbers. As a way toward a better understanding of their performance, it is useful to expand LGV2a and LGV2b by rewriting them as single time step schemes and putting them in the context of the second order weak Itô-Taylor expansion [Eq. (9)] as done for E2 in the previous section. In expanding these schemes, one should bear in mind that the time evolution is determined by differential equations of either pure diffusion type or pure drift type [see Eqs. (19)-(24) for their integrated forms]. This means that the Itô formula [cf. Eq. (6)] with $F=0$ in the operator $L^{(0)}$ should be used for the diffusive part and the third term in $L^{(0)}$, whereas $L^{(j)}(j \geqslant 1)$ must be set to zero for the drift part.

Starting with LGV2b, the term $F^{(k)}(\hat{Y}+1 / 2 D F(\hat{Y}) \tau)$ in Eq. (23) can be rewritten as

$$
\begin{aligned}
& F^{(k)}\left(\hat{Y}+\frac{1}{2} D F(\hat{Y}) \tau\right) \\
& \quad=F^{(k)}(\hat{Y})+\sum_{j=1} D^{(j, j)} F^{(j)}(\hat{Y}) \frac{\partial}{\partial Y^{(j)}} F^{(k)}(\hat{Y}) \frac{\tau}{2}
\end{aligned}
$$

due to the pure drift step $\hat{Y}+1 / 2 D F(\hat{Y}) \tau$. Expanding $F^{(k)}(\hat{Y})$ to the lowest possible order in $\tau$ and $\Delta \widetilde{W}$, one gets

$$
\begin{aligned}
F^{(k)}(\hat{Y})= & F^{(k)}\left(Y_{n}\right)+\frac{1}{2} \sum_{j} D^{(j, j)} \frac{\partial^{2}}{\partial Y^{(j)^{2}}} F^{(k)}\left(Y_{n}\right) \frac{\tau}{2} \\
& +\sum_{j} b^{(j, j)} \frac{\partial}{\partial Y^{(j)}} F^{(k)}\left(Y_{n}\right) \widetilde{\Delta W^{(j)}} .
\end{aligned}
$$

Substituting Eqs. (26) and (25) into Eq. (23) and retaining terms up to second order in Eq. (24) give

$$
\begin{aligned}
Y_{n+1}^{(k)}= & Y_{n}^{(k)}+b^{k, k}\left(\widetilde{\Delta W_{n}^{(k)}}\right. \\
& \left.+\widetilde{\Delta W_{n+1 / 2}^{\prime(k)}}\right)+D^{(k, k)}\left(F^{(k)}\left(Y_{n}\right) \tau+\frac{1}{2} \sum_{i=1}^{N} D^{(i, i)} F^{(i)}\left(Y_{n}\right)\right. \\
& \times \frac{\partial}{\partial Y^{(i)}} F^{(k)}\left(Y_{n}\right) \tau^{2}+\frac{1}{2} \sum_{i=1}^{N} D^{(i, i)} \frac{\partial^{2}}{Y^{(i)}} F^{(k)}\left(Y_{n}\right) \frac{\tau^{2}}{2} \\
& \left.+\sum_{i=1}^{N} b^{(j, j)} \frac{\partial}{\partial Y^{(j)}} F^{(k)}\left(Y_{n}\right) \tau \widetilde{\Delta W_{n}^{(i)}}\right)
\end{aligned}
$$

which is in a form similar to the second order Itô-Taylor expansion. Evaluating the following ensemble average of the stochastic integrals in Eq. (27): 


$$
\begin{aligned}
& \left\langle\left({\widetilde{\Delta W_{n}^{(k)}}}^{(k)}{\widetilde{\Delta W_{n+1 / 2}^{\prime}}}^{(k)}\right) \tau{\widetilde{\Delta W_{n}}}^{(i)}\right\rangle_{t_{0}} \\
& \quad=\left\langle{\widetilde{\Delta W_{n}}}^{(k)} \tau{\widetilde{\Delta W_{n}}}^{(i)}\right\rangle_{t_{0}}+0=\frac{1}{2} \tau^{2} \delta_{k, i}
\end{aligned}
$$

one is led to discover that the expectation value of Eq. (B3) in Appendix B is satisfied also by Eq. (27). Similar arguments can be used to show that also the other expectation values in Appendix B are satisfied. Using the same procedure, it would be possible to rewrite LGV2a [cf. Eq. (21)] by obtaining a scheme identical to Eq. (9). One shall therefore conclude that both LGV2a and LGV2b are consistent with a weak second order Itô-Taylor scheme. Incidentally, we mention here that the third scheme presented in Ref. 6 (LGV2c) is identical to E2, for which the consistency with the second order Itô-Taylor scheme has been already proved.

\section{NUMERICAL RESULTS AND DISCUSSION}

The main result presented in the previous sections is the formal demonstration that in addition to $\mathrm{E} 2$ and PC2, also LGV2a and LGV2b are second order schemes consistent with a weak Itô-Taylor expansion. As for the computational cost, one would expect E2 and LGV2b to be the least expensive ones requiring only two force evaluations and one or two vectors of $N$ random numbers per time step for E2 and LGV2b, respectively. LGV2a is likely to be the most costly, necessitating four force evaluations.

In the following sections, the performances of the four second order schemes are compared using numerical simulation results. Our strategy is similar to the one employed in Refs. 7 and 18, where model systems were initially simulated to provide indications on the time step bias of each algorithm. Different from what was done previously, ${ }^{7,18}$ in this initial stage we decided to evaluate only the performance of SDE integration algorithms, obtaining results that are equivalent to variational Monte Carlo (VMC) simulations in the limit of $\tau \rightarrow 0$. This allows us to clearly distinguish between the component of the error due to the discrete approximation of the SDE and to the operator splitting formula employed in DMC. The numerical results for one of our model systems (harmonic oscillator) are also compared with the analytical formula derived by means of a symbolic integration program, providing further support for the theory presented in the previous section and our numerical data. Subsequently, a more thorough set of tests for the four schemes is presented using helium clusters doped with $\mathrm{Mg} .{ }^{19}$ In these tests, both VMC and DMC simulations were run on $\mathrm{MgHe}_{12}$ as representative case using a $\mathrm{Mg}-\mathrm{He}$ interaction potential derived from CCSDT calculations. ${ }^{20}$

\section{A. VMC tests}

LGV2a and LGV2b were implemented in their original form, with the deterministic integrals evaluated using the second order Runge-Kutta method. ${ }^{6}$ Because of this, a single simulation step using LGV2a is twice more expensive than one using LGV2b. E2 and PC2 algorithms were implemented following Eqs. (12) and (14) as suggested in Ref. 12.

The first test on a model system was conducted by simulating the three-dimensional (3D) Gaussian distribution

$$
\psi_{T}(r)=\exp \left(-\alpha r^{2}\right)
$$

with $\alpha=0.5$, the numerical VMC results for $\left\langle r^{-1}\right\rangle$ obtained from the simulations shown in Fig. 1. From this, it is evident that PC2 outperforms all other algorithms over a very wide range of time step. Interestingly, PC2 seems also to provide a better order of convergence than expected from the theoretical analysis proposed previously. However, this is not uncommon for simple models such as the harmonic oscillator and should not be taken as a sound evidence for a superquadratic behavior (vide infra). The improvement in performance provided by $\mathrm{PC} 2$ is particularly striking when compared with LGV2b: PC2 allows us to use a time step roughly an order of magnitude larger than LGV2b, obtaining similar systematic errors. In turn, this provides a clear algorithmic advantage due to the faster data decorrelation obtained when using large time steps. Despite the somewhat limited improvement provided over LGV2b, E2 nevertheless appears as a suitable candidate to substitute for LGV2b due to the similar cost per time step.

The simplicity of the harmonic oscillator allows one to derive the analytical form for the eigenfunction with unit eigenvalue of the integral equation associated with each of the four tested algorithms. In other words, it is possible to obtain the stationary distribution $f(x, t)$ satisfying

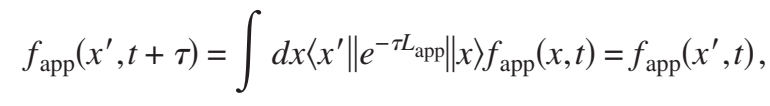

where $L_{\text {app }}$ represents the approximate Fokker-Planck operator associated with each algorithm and $f_{\text {app }}(x, t)$ is the associated stationary distribution. Whereas obtaining analytical results for LGV2a and LGV2b was found to be straightforward, ${ }^{7,18}$ it was less so for E2 and PC2. Indeed, one needs to take into account that the same Gaussian displacement is used during all predictor/corrector moves and that a convolution is necessary to feedback the predicted position into the correction step. In all four cases, we found that it was possible to Taylor expand the stationary distributions obtained from Eq. (30) with respect to $\tau$, obtaining the leading components of the error. The latter read

$$
\begin{aligned}
& f_{\mathrm{LGV} 2 \mathrm{a}}(x, t)=e^{-2 \alpha x^{2}}\left(1-\alpha^{3} x^{2} \tau^{2}+O\left(\tau^{3}\right)\right), \\
& f_{\mathrm{LGV} 2 \mathrm{~b}}(x, t)=e^{-2 \alpha x^{2}}\left(1+4 \alpha^{3} x^{2} \tau^{2}+O\left(\tau^{3}\right)\right) \\
& f_{\mathrm{E} 2}(x, t)=e^{-2 \alpha x^{2}}\left(1-2 \alpha^{3} x^{2} \tau^{2}+O\left(\tau^{3}\right)\right) \\
& f_{\mathrm{PC} 2}(x, t)=e^{-2 \alpha x^{2}}\left(1+2 \alpha^{4} x^{2} \tau^{3}+O\left(\tau^{4}\right)\right)
\end{aligned}
$$

clearly indicating the second order of LGV2a, LGV2b, and $\mathrm{E} 2$, as well as the third order behavior for PC2. Moreover, the direct comparison between the previous equations and the results in Fig. 1 also points out the good agreement, in terms of the relative error, between analytical and numerical results.

To obtain a more stringent test of the SDE schemes, we also employed a 3D trial wave function reminiscent of the model functions used to simulate He droplets, namely, 


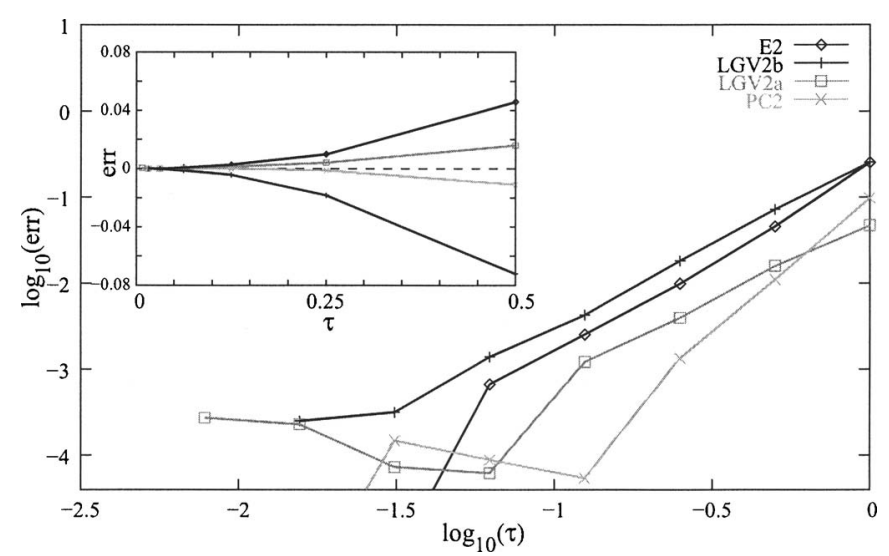

FIG. 1. Error in $\left\langle r^{-1}\right\rangle\left[\operatorname{bohr}^{-1}, \log _{10}(|\operatorname{err}|)\right]$ for the harmonic oscillator ground state $\exp \left(-\alpha r^{2}\right)$ as a function of time step [a.u., $\left.\log _{10}(\tau)\right]$ for schemes E2 $(\diamond)$, PC2 $(\times)$, LGV2a $(\square)$, and LGV2b $(+)$. Errors below $10^{-3.2}$ are dominated by statistical fluctuations.

$$
\psi_{T}(r)=\exp \left(-a r-b / r^{5}\right)
$$

where $a=10$ and $b=2.5$. This choice of parameters is consistent with the one obtained after optimization for systems such as $\mathrm{AgHe}_{n}(n=2-8),{ }^{21}$ and are representative of $\mathrm{He}$ strongly bound to a heavy attractive center. The results of our simulations are presented in Fig. 2. For $\tau<10^{-2.4}$ (0.004) a.u., the performance of the algorithms closely resembles the one discussed for the harmonic oscillator. However, for larger $\tau$ both E2 and PC2 clearly outperform LGV2a and LGV2b. More interestingly, a sudden change in behavior is seen for LGV2a and LGV2b, deviating strongly from the expected quadratic convergence of the error. This clearly indicates a narrower range of convergence for LGV2a and LGV2b, an unpleasant feature apparently not shared by E2 and PC2 whose range of convergence is almost an order of magnitude wider. The consequences of these findings on the performance of the algorithms will be discussed in the following.

Although preliminary, some conclusions can be drawn from the presented results. First, it is evident that E2 outperforms the equally economical LGV2b, while at the same time providing a better stability and a wider convergence

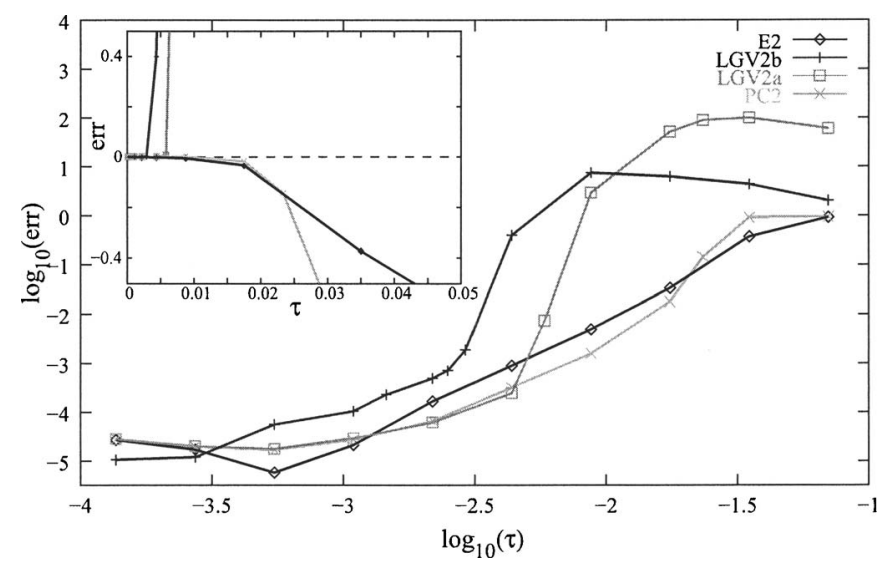

FIG. 2. Error in $\left\langle r^{-1}\right\rangle\left[\mathrm{bohr}^{-1}, \log _{10}(|\mathrm{err}|)\right]$ for the trial wave function in Eq. $(35)$ as a function of time step [a.u., $\left.\log _{10}(\tau)\right]$ for schemes E2 $(\diamond), \mathrm{PC} 2(\times)$, LGV2a $(\square)$, and LGV2b $(+)$. Errors below $10^{-4}$ are dominated by statistical fluctuations.

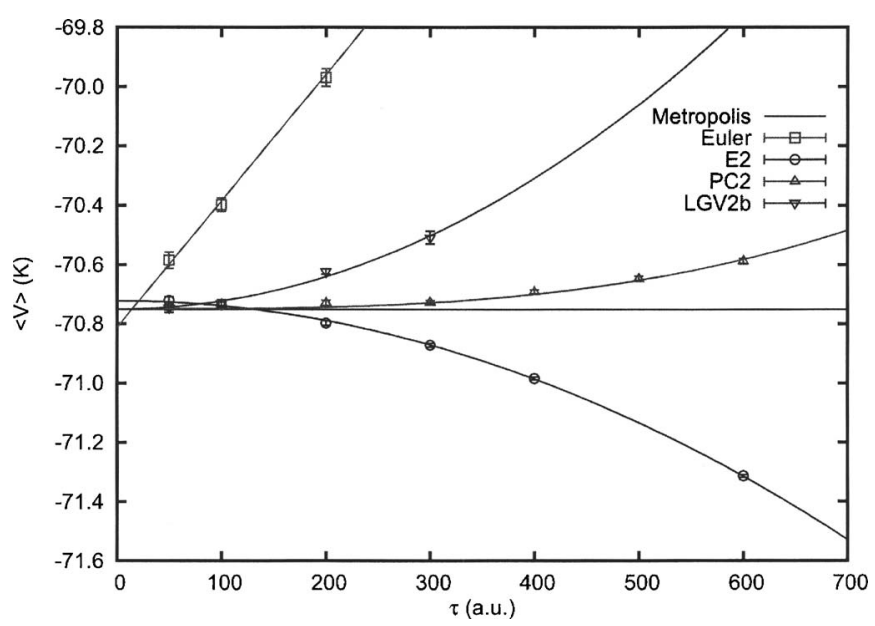

FIG. 3. Expectation value of the interaction potential $\langle V\rangle(\mathrm{K})$ as a function of the time step (a.u.) for a VMC simulation of $\mathrm{MgHe}_{12}$. The Metropolis result represents the limiting value for $\tau \rightarrow 0$ that should be obtained by all simulation algorithms. The continuous lines are linear (Euler), quadratic (E2 and LGV2b), and cubic (PC2) fits to the numerical results.

range. Second, the time step bias for PC2 is found to be smaller than for LGV2a, the latter being more expensive due to an additional force evaluation. So, the predictor-corrector schemes based on the Itô-Taylor expansion appear as suitable candidates to substitute for the second order exponential formulas proposed in Ref. 6. Bearing all this in mind, we decided to avoid any further testing on LGV2a because of the high cost-performance ratio manifested during the model simulations and refer the reader to Ref. 7 for a relative comparison between this algorithm and LGV2b.

As a concluding test for the performance of the SDE schemes in VMC simulations, the sampling of the ground state distribution for $\mathrm{MgHe}_{12}$ was carried out using E2, PC2, LGV2b, and the Euler scheme in Eq. (4). The form of the wave function was taken from Ref. 19 and the parameters were slightly modified to generate a less smooth local energy. This choice increases the time step bias for the average local energy, making also more apparent shortcomings in the simulation algorithms. The mean values of the interaction potential as a function of the time step are shown in Fig. 3, together with the "exact" value obtained from a standard Metropolis simulation. The results for this system present a behavior similar to the one shown by the two model systems, with the Euler algorithm converging linearly with respect to $\tau$. Both LGV2b and E2 appear to have a quadratic convergence to the $\tau \rightarrow 0$ limit, whereas the PC2 results could be properly fitted only using a cubic function. Once again, PC2 is found to perform better than the other schemes, allowing to use a time step roughly four times larger than LGV2b and twice as large than E2 while producing similar systematic errors. We also found that the simulations run with both $\mathrm{Eu}-$ ler and LGV2b became unstable for $\tau>300$ a.u., whereas E2 and PC2 appeared to be more stable, perhaps owing to a better control exerted on sudden changes in the quantum force, with the simulations becoming unstable only for $\tau>600$ a.u. 


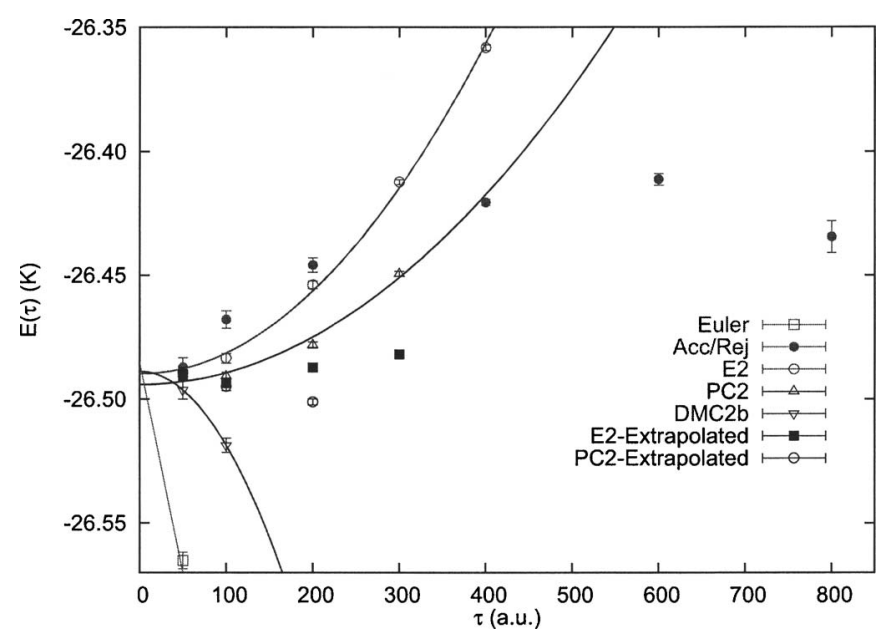

FIG. 4. Expectation value of the local energy $\left\langle E_{L}\right\rangle(\mathrm{K})$ as a function of the time step (a.u.) for DMC simulations of $\mathrm{MgHe}_{12}$. The continuous lines are linear (Euler) and quadratic (E2, PC2, and DMC2b) fits to the numerical results. The results for the Euler scheme with acceptance/rejection (Acc/Rej) step were not fitted due to the lack of a theoretical justification for the order of this algorithm. The extrapolated $(\tau=0)$ values have been obtained assuming a second order behavior for $\left\langle E_{L}\right\rangle$ as a function of $\tau$.

\section{B. DMC tests}

The good performances of both E2 and PC2 in sampling the trial distribution $\psi_{T}^{2}$ (Figs. 1 and 2) suggest that it may be possible to reduce the DMC time step error in the simulation of bosonic clusters. Before describing our DMC results, it is perhaps worth stressing once more that the order (hence the error) of the simulations is somewhat limited by the choice of using Eq. (3) as an approximation of the exact propagator. In other words, one should not expect the order of the time step bias to be higher than second even though the exact simulation of the Fokker-Planck equation was possible, an outcome due to the neglect of double commutators (e.g., $[T,[V, T]])$ in the exponential formulas. ${ }^{4}$ Nevertheless, accurate integrators for the Langevin equation may result in a substantially lower time step bias and, perhaps, in a more robust algorithm with a better defined order of convergence, as suggested by the VMC simulations on $\mathrm{MgHe}_{12}$.

Our test case for DMC is, again, provided by $\mathrm{MgHe}_{12}$, for which precise results could be obtained at low computational cost. All simulations were run by sampling, at least, 3500 blocks composed by 500 steps each, and with a target population of 5000 walkers. The results have been checked for any residual presence of serial correlation between blocks and the associate standard error corrected accordingly. Figure 4 presents the average value of the local energy as a function $\tau$ in the simulations. In this case, the improvement provided by E2 and PC2 over Euler and DMC2b [obtained using LGV2b in Eq. (3)] in terms of the time step bias is clearly evident. Specifically, PC2 reduces the time step bias by roughly a factor of 3 with respect to $\mathrm{E} 2$ and by roughly a factor of 6 with respect to DMC2b. Figure 4 also presents results obtained using the standard DMC algorithm with acceptance/rejection step, ${ }^{22}$ as commonly implemented using Eq. (4). In this case, the expected linear behavior is modified by the additional Metropolis step, which, despite producing a smaller time step bias than the Euler algorithm, induces a trend of $\left\langle E_{L}\right\rangle$ versus $\tau$ difficult to interpret theoretically. We consider this to be a serious drawback, which limits the possibility of estimating the systematic error of the simulations or of extrapolating their results. Besides, the comparison between the standard DMC algorithm and second order PC2 scheme indicates a smaller bias for the latter if $\tau<400$ a.u., suggesting PC2 as a very useful alternative despite the lack of the Metropolis correction step. The latter, however, appears to have a positive effect on the stability of the simulations. In fact, these are found to be stable over a wider range of $\tau(0-800$ a.u. $)$ than in the case of DMC2b (0-100 a.u.), E2 (0-400 a.u.), or PC2 (0-300 a.u.).

The robust order of E2 and PC2 (when compared with the standard DMC scheme) and their wide range of quadratic convergence provide us with the additional possibility of exploiting Richardson's extrapolation method ${ }^{23}$ to improve the accuracy of the numerical results a posteriori. ${ }^{24}$ The results obtained by extrapolating the data as suggested in Ref. 24 are therefore presented in Fig. 4 and provide a substantially reduced time step bias. A similar behavior was obtained for other observables (e.g., the average interaction potential), once again a direct consequence of the robust second order behavior of E2 and PC2.

\section{CONCLUSIONS}

In this work, the performances of the two algorithms E2 and PC2 when used in importance sampled DMC simulations of bosonic clusters have been theoretically scrutinized and numerically assessed. By numerically comparing E2and PC2-derived DMC schemes with several other algorithms, ${ }^{6,2}$ we found that the first two schemes provided smaller time step bias than split-operator derived algorithms using a larger number of force evaluations. In particular, PC2 presents excellent performances in comparison to $\mathrm{LGV} 2 \mathrm{a} / \mathrm{b}$ Ref. 6 and we consider it as the best available second order algorithm, among the ones we tested, in the context of DMC simulations using Eq. (3).

The interplay between algorithm efficiency and time step error becomes extremely important when simulating systems larger than $\mathrm{MgHe}_{12}$. Due to the increased cost, it is quite common to avoid the extrapolation to $\tau \rightarrow 0$ by choosing a maximum acceptable discretization error for some property of the system and selecting the time step accordingly. ${ }^{10}$ When using this protocol with bosonic clusters, it becomes readily apparent that the larger the time step, the smaller is the standard error for a given simulation length. ${ }^{10}$ This is indicated in Fig. 5, which shows the simulation standard error $\sigma(\tau)$ of $\left\langle E_{L}\right\rangle$ for $\mathrm{MgHe}_{12}$ as a function of $\tau .^{25}$ Independent of the algorithm, $\sigma(\tau)$ appears to be proportional to $\tau^{-1 / 2}$ (represented by the continuous lines in Fig. 5) for $50 \leqslant \tau$ $\leqslant 400$ a.u., stressing the fact that an increase in the time step by a factor of 2 should improve the algorithm efficiency by the same amount. Assuming that an acceptable systematic error for $\left\langle E_{L}\right\rangle$ is $0.1 \%$, from Figs. 4 and 5 it is apparent that PC2 may improve the efficiency of DMC simulations by a factor of 10 over the simpler Euler algorithm and by a factor of roughly 3 with respect to DMC2b. Taking into account also the additional cost represented by the larger number of 


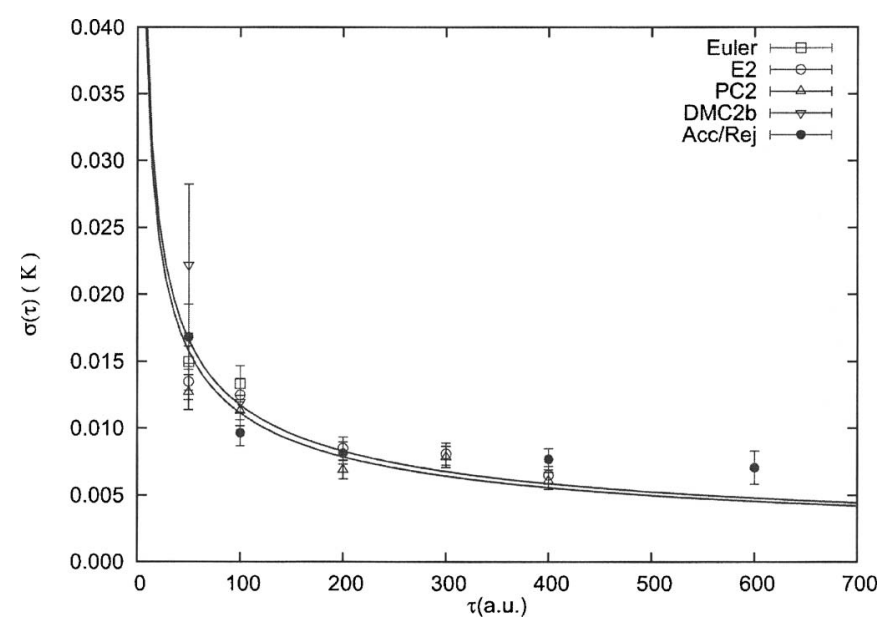

FIG. 5. Decorrelated standard error for the expectation value of the local energy $\left\langle E_{L}\right\rangle(\mathrm{K})$ as a function of the time step $\tau$ (a.u.) for DMC simulations of $\mathrm{MgHe}_{12}$. The continuous lines are fitted to the E2 and Acc/Rej results using the functional form $\sigma(\tau)=a \tau^{-1 / 2}$.

force evaluation required, the advantage provided by PC2 is somewhat reduced, making it roughly three times more efficient than the Euler scheme and almost twice as efficient than DMC2b. Compared with the algorithm using the acceptance/rejection step, PC2 allows to increase the time step by a factor of 1.5-2, an improvement that is nullified by the larger computational cost per step. It is, however, important to stress that both E2- and PC2-derived DMC schemes allow the extrapolation. ${ }^{23}$ of large time step data with a net gain in the overall efficiency of the simulation protocol. As an example, we point out (see Fig. 4) that the extrapolation of E2 data at $\tau=300,400$ a.u. substantially improves the accuracy, reducing the time step bias by a factor of 15 (e.g., see $\tau=100$ a.u.) and the total computational cost by a factor of 2 . So, despite the necessity of running two simulations, the extrapolation makes the usage of E2 and PC2 advantageous also with respect to the algorithm employing acceptance/ rejection.

As a final comment on the algorithm performances, it is important to stress that both E2- and PC2-derived schemes were found to be more robust than the simpler Euler and DMC2b, therefore allowing to use longer time steps. A similar, if not better, stability is also featured by the commonly used algorithm employing a rejection step, probably due to the control exerted by the Metropolis step on the final position of the walker. This may prove beneficial also for E2- or PC2-based schemes, but we found it difficult to provide the analytical form of the transition matrix simulated by E2 and PC2, which is needed to compute the acceptance probability.

We conclude this discussion by pointing out that the increased performances provided by E2 and PC2 pave the way for further improvements and for their usage in different contexts. For instance, one may be able to extrapolate the results of the integration algorithm "on the fly" producing, with the help of the Feynman-Kac formula, ${ }^{12}$ a DMC algorithm showing at least a third order behavior. As an alternative application of E2 and PC2, we mention the reptation quantum Monte Carlo (RQMC) algorithm ${ }^{26}$ which is used to compute imaginary-time correlation functions. Also in this case, the focus must be placed on accurate weak schemes as thoroughly examined in Ref. 27, and both E2 and PC2 may be used in RQMC to reduce the number of segments in the "reptile". This is likely to have a strong impact on the simulation efficiency due the quadratic scaling of the cost with the reptile length. In this respect, we are planning to implement a new version of RQMC employing PC2 to compute response properties for large doped helium clusters. Needless to say, if only unbiased (i.e., from $\psi_{0}^{2}$, not $\psi_{T} \psi_{0}$ ) expectation values were required, other DMC-based algorithms ${ }^{28-30}$ could be used instead of RQMC. In this case, we would expect E2/PC2 to be easily integrated in those approaches, with only minor modifications in the part of the code carrying out the DMC evolution starting from $\psi_{T}^{2}$. Because the latter is usually sampled using unbiased Metropolis schemes before the DMC propagation, the lack of acceptance/rejection step in E2/PC2 introduces no additional theoretical difficulties in obtaining unbiased expectation values.

\section{ACKNOWLEDGMENTS}

One of the authors (M.M.) acknowledges an EPSRC Advanced Research Fellowship (GR/R77803/02) and the EPSRC Grant No. EP/C528301/1.

\section{APPENDIX A: SECOND ORDER ITÔ-TAYLOR EXPANSION}

With the quantum force expanded in the form of Eq. (6) and inserted into Eq. (5) we obtain

$$
Y_{t}^{(k)}=Y_{n}^{(k)}+D^{(k, k)} F^{(k)}\left(Y_{n}\right) \int_{t_{n}}^{t_{n+1}} d s+b^{(k, k)} \int_{t_{n}}^{t_{n+1}} d W^{(k)}+R
$$

where the remainder is

$$
\begin{aligned}
R= & D^{(k, k)} \int_{t_{n}}^{t_{n+1}} \int_{0}^{s} L^{(0)} F^{(k)}\left(Y_{z}\right) d z d s \\
& +D^{(k, k)} \sum_{j}^{N} \int_{t_{n}}^{t_{n+1}} \int_{t_{n}}^{s} L^{(j)} F^{(k)}\left(Y_{z}\right) d W_{z}^{(j)} d s .
\end{aligned}
$$

By setting the remainder $R$ to zero in Eq. (A1), a scheme accurate to first order in time step is obtained, and this can be easily identified with Eq. (4). The second order weak ItôTaylor scheme is obtained by substituting Eq. (6) for the functions $L^{(0)} F^{(k)}\left(Y_{z}\right)$ and $L^{(j)} F^{(k)}\left(Y_{z}\right)$ in Eq. (A2) and it takes the form 


$$
\begin{aligned}
Y_{n+1}^{(k)}= & Y_{n}^{(k)}+D^{(k, k)} F^{(k)}\left(Y_{n}\right) \int_{t_{n}}^{t_{n+1}} d s+D^{(k, k)} L^{(0)} F^{(k)}\left(Y_{n}\right) \\
& \times \int_{t_{n}}^{t_{n+1}} \int_{t_{n}}^{s} d z d s+D^{(k, k)} \sum_{j=1}^{N} L^{(j)} F^{(k)}\left(Y_{n}\right) \\
& \times \underbrace{\int_{t_{n}}^{t_{n+1}} \int_{t_{n}}^{s} d W_{z}^{(j)} d s}_{=I_{(j, 0)}}+b^{(k, k)} \underbrace{\int_{t_{n}}^{t_{n+1}} d W^{(k)}}_{=I_{(k)}}
\end{aligned}
$$

In using Eq. (A3) for a numerical scheme, the deterministic integrals are exactly evaluated as

$$
\int_{t_{n}}^{t_{n+1}} d s=\tau_{n}, \quad \int_{t_{n}}^{t_{n+1}} \int_{t_{n}}^{s} d z d s=\frac{\tau_{n}^{2}}{2}
$$

where $\tau_{n}=t_{n+1}-t_{n}$. The evaluation of the stochastic integrals in a weak Itô expansion ${ }^{16}$ need not be as strict as for their deterministic counterparts. Actually, it is only necessary to fulfill a specific set of conditions for the conditional expectation values of $I_{(k)}$ and $I_{(j, 0)}$ in order to have a second order scheme (these conditions are listed in Appendix B). In other words, $I_{(k)}$ and $I_{(j, 0)}$ can be substituted with two alternative stochastic integrals $\tilde{I}_{(k)}, \tilde{I}_{(j, 0)}$ provided that the conditional expectation value of the latter differs from those listed in Appendix B [Eqs. (B1)-(B4)] by, at most, $K \tau^{3}(K>0)$. This is the case if the stochastic integrals are evaluated as

$$
\tilde{I}_{(k)}=\Delta W_{n}^{(k)}, \quad \tilde{I}_{(j, 0)}=\frac{1}{2} \tau_{n} \Delta W_{n}^{(j)},
$$

where $\Delta W_{n}^{(j)}$ can be generated as a Gaussian random variable $N\left(0, \tau_{n}\right)$ (see Ref. 12, p. 225). Rewriting Eq. (A3) using Eqs. (A4) and (A5), we get the simplified second order weak ItôTaylor scheme [Eq. (9)].

\section{APPENDIX B: MULTIPLE ITÔ INTEGRALS}

Below, we list the conditional expectation values of the stochastic integrals $\left\{I_{(k)}, I_{(j, 0)}\right\}$ in Eq. (A3). These are of relevance for the second order Itô-Taylor expansion ${ }^{12}$

$$
\begin{aligned}
& \left\langle I_{(i)}\right\rangle_{t_{0}}=\left\langle I_{(i)} I_{(j)} I_{(k)}\right\rangle_{t_{0}} \\
& \quad=\left\langle I_{(i, 0)} I_{(j)} I_{(k)}\right\rangle_{t_{0}}=\left\langle I_{(i)} I_{(j)} I_{(k)} I_{(l)} I_{(m)}\right\rangle_{t_{0}}=0, \\
& \left\langle I_{(i)} I_{(j)}\right\rangle_{t_{0}}=\tau \delta_{i, j}, \\
& \left\langle I_{(i)} I_{(j, 0)}\right\rangle_{t_{0}}=\frac{1}{2} \tau^{2} \delta_{i, j},
\end{aligned}
$$

$$
\begin{aligned}
& \left\langle I_{(i)} I_{(j)} I_{(k)} I_{(l)}\right\rangle_{t_{0}} \\
& \quad= \begin{cases}3 \tau^{2} & \text { if } i=j=k=l \\
\tau^{2}, & \{i, j, k, l\} \text { consists of two distinct pairs } \\
& \text { of identical numbers } \\
0 & \text { otherwise }\end{cases}
\end{aligned}
$$

where $\delta_{i, j}$ is a Kronecker delta, $(i, j, k, l, m) \in\{1,2, \ldots, N\}$, and the subscript $t_{0}$ means that the integrals in each expectation value are evaluated from the same initial instant.

${ }^{1}$ J. B. Anderson, J. Chem. Phys. 63, 1499 (1975).

${ }^{2}$ W. M. C. Foulkes, L. Mitas, R. J. Needs, and G. Rajagopal, Rev. Mod. Phys. 73, 33 (2001).

${ }^{3}$ M. H. Kalos, D. Levesque, and L. Verlet, Phys. Rev. A 9, 2178 (1974).

${ }^{4}$ M. Suzuki, J. Math. Phys. 26, 601 (1985).

${ }^{5}$ There are, however, situations in which the second order behavior is negated by the failure to fulfill some conditions, e.g., the presence of nonanalytic points in the ground state wave function.

${ }^{6}$ S. A. Chin, Phys. Rev. A 42, 6991 (1990).

${ }^{7}$ H. A. Forbert and S. A. Chin, Phys. Rev. B 63, 144518 (2001).

${ }^{8}$ E. Helfand, Bell Syst. Tech. J. 58, 2289 (1979).

${ }^{9}$ J. B. Anderson, J. Chem. Phys. 82, 2662 (1985).

${ }^{10}$ S. Chiesa, M. Mella, G. Morosi, and D. Bressanini, J. Chem. Phys. 119, 5601 (2003).

${ }^{11}$ B. Øksendal, Stochastic Differential Equations: An Introduction with Applications, 5th ed. (Springer, Berlin, 1998).

${ }^{12}$ P. E. Kloeden and E. Platen, Numerical Solution of Stochastic Differential Equations (Springer, Berlin, 1992).

${ }^{13}$ E. Platen, Math. Comput. Simul. 38, 69 (1995).

${ }^{14}$ H. P. Breuer, U. Dorner, and F. Petruccione, Comput. Phys. Commun. 132, 30 (2000).

${ }^{15}$ Itô formula is the differential rule for a function in Itô calculus; the integrated form is used.

${ }^{16}$ A. Tocino and R. Ardanuy, J. Comput. Appl. Math. 138, 219 (2002).

${ }^{17}$ W. H. Press, S. A. Teukolsky, and B. P. Flannery, Numerical Recipes in C (Cambridge University Press, Cambridge, 1992).

${ }^{18}$ M. Mella, G. Morosi, and D. Bressanini, Phys. Rev. E 61, 2050 (2000).

${ }^{19}$ M. Mella, G. Calderoni, and F. Cargnoni, J. Chem. Phys. 123, 054328 (2005).

${ }^{20}$ R. J. Hinde, J. Phys. B 36, 3119 (2003).

${ }^{21}$ M. Mella, M. C. Colombo, and G. Morosi, J. Chem. Phys. 117, 9695 (2002).

${ }^{22}$ P. J. Reynolds, D. M. Ceperley, B. J. Alder, and W. A. Lester, J. Chem. Phys. 77, 5593 (1982).

${ }^{23}$ P. E. Koeden, E. Platen, and N. Hofmann, SIAM (Soc. Ind. Appl. Math.) J. Numer. Anal. 32, 1919 (1995).

${ }^{24}$ L. Brualla, K. Sakkos, J. Boronat, and J. Casulleras, J. Chem. Phys. 121, 636 (2004).

${ }^{25}$ This is obtained from simulations sampling an identical number of configurations and estimating the standard error using the blocking algorithm Ref. 31.

${ }^{26}$ S. Baroni and S. Moroni, Phys. Rev. Lett. 82, 4575 (1999).

${ }^{27}$ P. Håkansson, L. Persson, and P.-O. Westlund, J. Chem. Phys. 117, 8634 (2002).

${ }^{28}$ R. N. Barnett, P. J. Reynolds, and W. A. Lester, J. Comput. Phys. 96, 258 (1991).

${ }^{29}$ K. S. Liu, M. H. Kalos, and G. V. Chester, Phys. Rev. A 10, 303 (1974).

${ }^{30}$ M. H. Kalos and F. Arias de Saavedra, J. Chem. Phys. 121, 5143 (2004).

${ }^{31}$ H. Flyvieng and H. G. Peterson, J. Chem. Phys. 91, 461 (1989). 